\title{
Corrigendum: Identification of the phytosphingosine metabolic pathway leading to odd-numbered fatty acids
}

Natsuki Kondo, Yusuke Ohno, Maki Yamagata, Takashi Obara, Naoya Seki, Takuya Kitamura, Tatsuro Naganuma \& Akio Kihara

Nature Communications 5:5338 doi: 10.1038/ncomms6338 (2014); Published 27 Oct 2014; Updated 2 Apr 2015

In this Article, there are errors in the labelling of the thin layer chromatogram in Fig. 6e. The two lanes labelled ' + ' should have been labelled '-', and the two lanes labelled '-' should have been labelled ' + '. The correct version of this figure follows. 


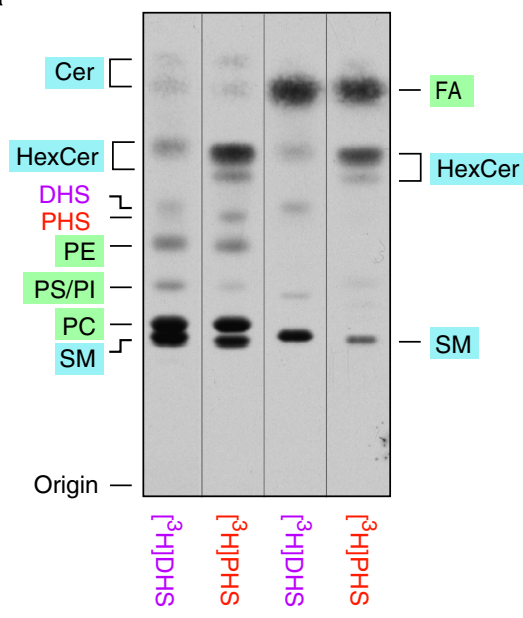

Alkaline

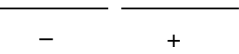

C

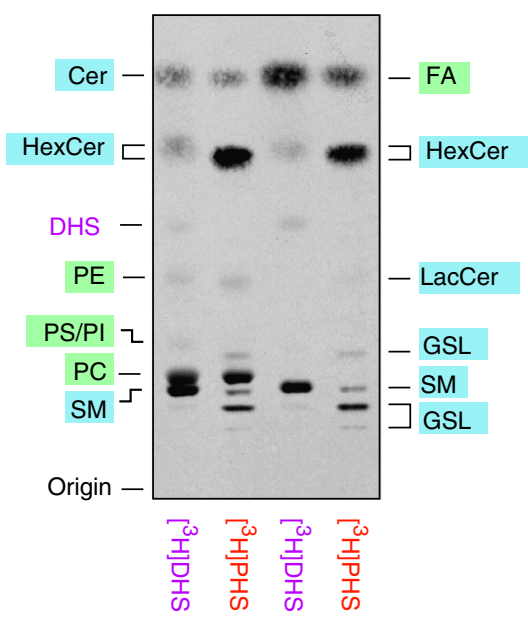

Alkaline $-\overline{+}$ b
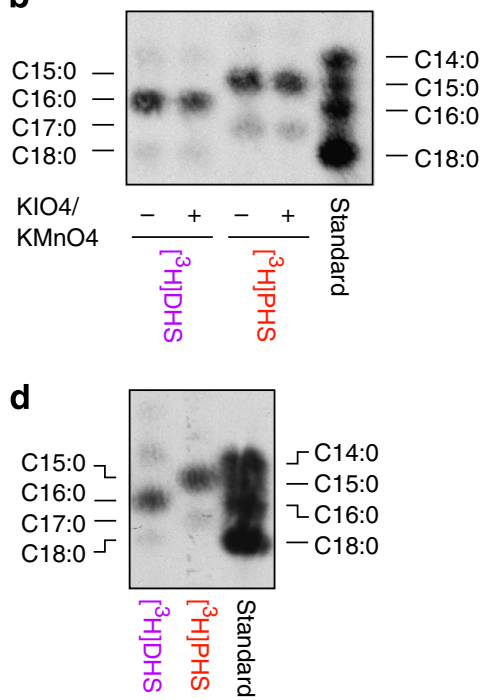

e

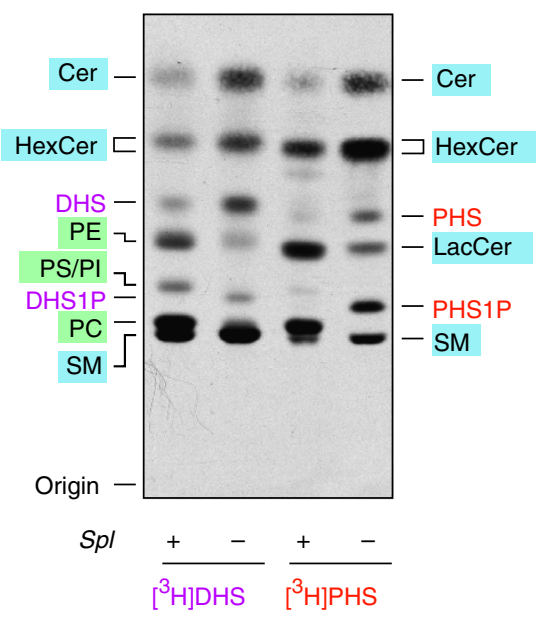

Figure 6 